\title{
REVIEW ARTICLE OPEN Evidence for a causal role by mouse mammary tumour-like virus in human breast cancer
}

\author{
James S. Lawson ${ }^{1 *}$ and Wendy K. Glenn ${ }^{1}$
}

\begin{abstract}
We have reviewed the evidence relevant to mouse mammary tumour viruses (MMTV) and human breast cancer. The prevalence of MMTV- like gene sequences is 15 -fold higher in human breast cancer than in normal human breast tissue controls and is present in up to $40 \%$ of human breast cancers. MMTV-like gene sequences can be identified in benign breast tissues 1-11 years before the development of positive MMTV-like breast cancer in the same women. The prevalence of MMTV antibodies in sera from women with breast cancer is 5-fold higher than in normal women. MMTV can infect human breast epithelial cells and integrate at random into the human genome located in those cells. MMTV-like gene sequences are present in human milk from normal lactating women and with increased prevalence in milk from women at risk of breast cancer. MMTV-like virus associated human breast cancer has strikingly similar features to MMTV-associated mouse mammary tumours. These features include almost identical nucleotide sequences and structure of the MMTV genome, histology, superantigen expression, MMTV infection of B and T lymphocytes and hormone dependence. MMTV-like gene sequences have also been identified in dogs, cats, monkeys, mice and rats. Saliva has been identified as the most plausible means of transmission from human to human and possibly from dogs to humans. The evidence meets the classic causal criteria. A causal role for MMTV-like viruses in human breast cancer is highly likely.
\end{abstract}

npj Breast Cancer (2019)5:40; https://doi.org/10.1038/s41523-019-0136-4

\section{INTRODUCTION}

We have reviewed the evidence relevant to the underlying causes of breast cancer. In our view, there is now sufficient evidence to conclude that a mouse mammary tumour-like virus (MMTV-like) is probably causal in a significant number of breast cancers. The evidence was assessed by using an extended version of the classic Austin Bradford Hill causal criteria. ${ }^{1}$ He established the following criteria-strength of association, consistency, specificity, temporality, biological gradient, plausibility, coherence, experiment and analogy. We have included additional criteria to address more current scientific developments in studies of oncoviruses. These are-the presence of viral genetic material in cancer tissues but rarely in normal tissues, the virus being capable of transforming normal cells into malignant cells, a potential viral oncogenic mechanism and the potential means of viral transmission. There are differences in the importance of each criterion. With respect to viruses and cancer the presence of viral genetic material prior to the development of same virus positive cancer, the strength of association and a significant odds ratio between cancer and normal tissues, are of special importance.

\section{Background}

In 1936, John Bittner discovered a pathogenic agent, which he called a "milk factor', which could be transmitted by milk from mouse mothers with mammary tumours to their mouse pups, who as adults, later developed mammary tumours. ${ }^{2}$ A decade later, Samuel Graff and colleagues identified viral particles in mouse milk which could cause mammary cancer when injected intraperitoneally into laboratory strains of mice. ${ }^{3}$ These virus particles were later identified as a retroviral RNA virus, which became known as mouse mammary tumour virus. ${ }^{4}$ A period of intensive studies into the biology of MMTV and its possible role in human breast cancer, financed by President Richard Nixon's "war on cancer", followed between 1970 and 1985. However, although there were numerous sporadic reports, no definitive links between MMTV and human breast cancer were convincingly identified. As a consequence of this, but also the discovery of human endogenous retroviruses (HERVs), which are similar to currently known exogenous viruses and which many thought accounted for these sporadic reports, interest linking MMTV and breast cancer waned. As a result of the development of sophisticated new techniques such as polymerase chain reaction (PCR), which have enormously increased the sensitivity of detection, there has been renewed interest in the search for a potential role of MMTV in human breast cancer.

\section{Search strategy and selection criteria}

The main source of publications cited in this review is PubMed Central using the search terms breast cancer, mouse mammary tumour virus and mouse mammary tumours-from 1936 to 2019. The publications listed in Table 1, which report the results of case control studies of MMTV in human breast cancer were selected because of the quality of the methods used. Studies which did not investigate normal breast tissues or investigated expression of non-env gene sequences or where the primers used were not traceable, were excluded.

\section{STRENGTH AND CONSISTENCY OF ASSOCIATION BETWEEN MMTV AND BREAST CANCER}

Identification of MMTV-like gene sequences in human breast cancer

There has been reasonably consistent identification of MMTV-like gene sequences in human breast cancers as compared to the extremely low levels of identification in normal and benign breast tissues. Prior to the widespread use of PCR, MMTV-related

\footnotetext{
${ }^{1}$ School of Biotechnology and Biomolecular Science, University of New South Wales, Sydney, NSW, Australia. *email: James.lawson@unsw.edu.au
} 


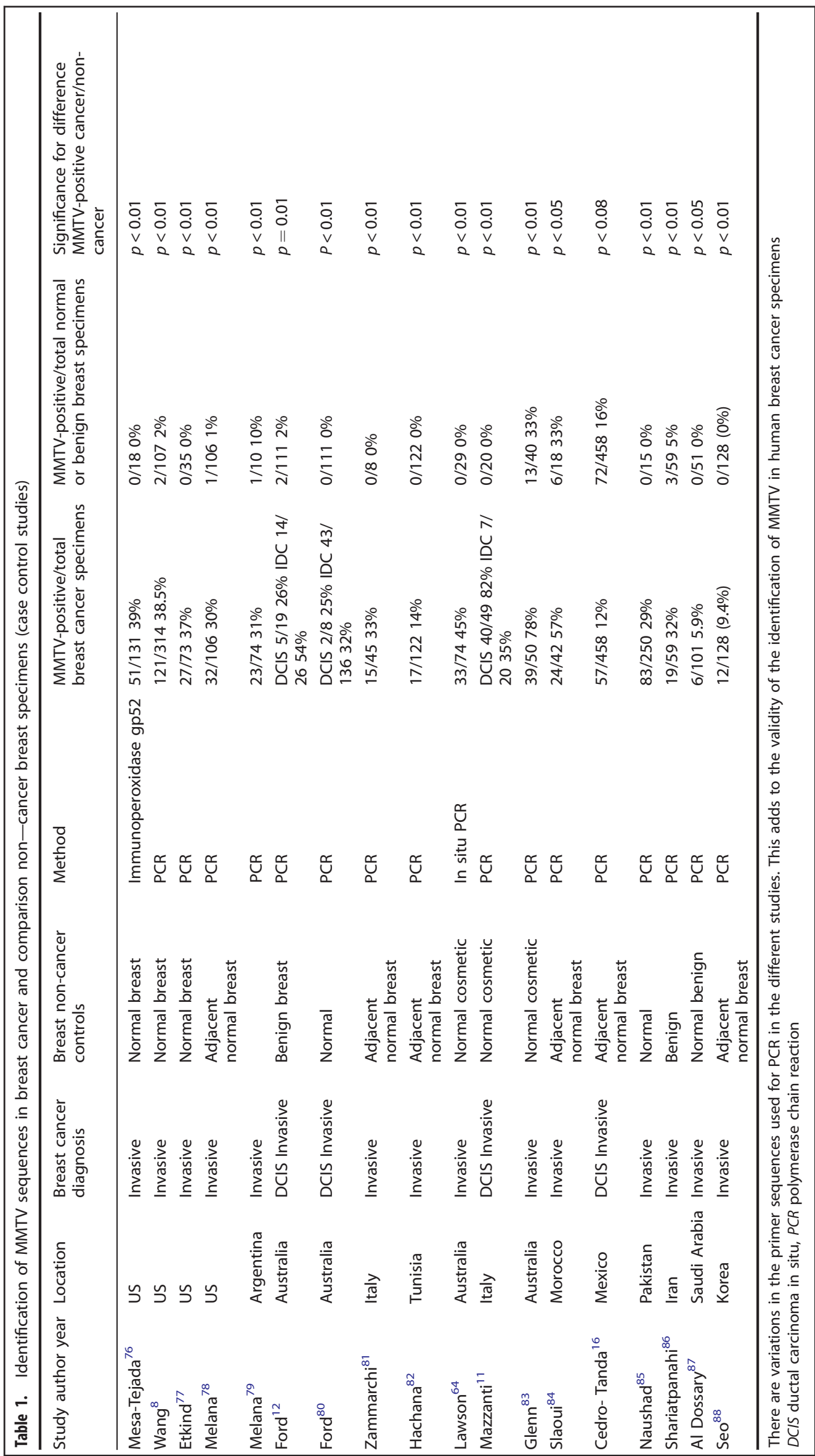




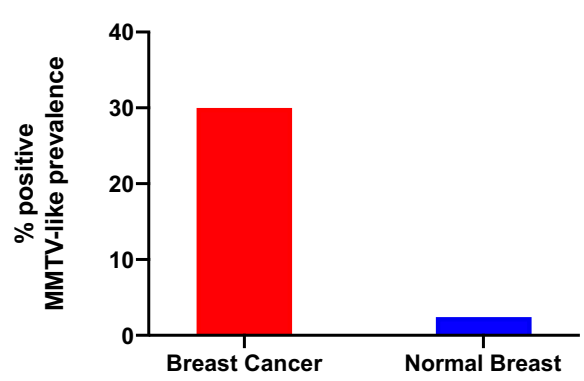

Fig. 1 Prevalence of MMTV-like gene sequences in invasive human breast cancers compared to normal human breast tissue controls (based on Table 1)

sequences in human breast cancer cells were identified by hybridisation techniques. ${ }^{5}$ In early studies Callahan and coworkers were able to identify MMTV on Southern blots hybridized to both a full length MMTV specific probe, as well as to probes specific for the typical structures of a retrovirus, gag-pol and env, in human normal and breast tumour DNA. ${ }^{5}$ Using hybridisation methods Szakacs \& Moscinski ${ }^{6}$ also identified DNA sequences homologous to the entire MMTV provirus using LTR- long terminal repeat, gag, pol and env probes in 7 (13\%) of 52 human breast cancers. ${ }^{6}$

Until the mid-1990's, there had been a controversy around the distinction of MMTV from human endogenous retroviruses (HERV) using such hybridization techniques, since these two retrovirus families share a high degree of nucleotide homology in certain regions which might lead to positive signals. ${ }^{7}$

\section{PCR Studies}

Using PCR techniques directed at a $660 \mathrm{bp}$ highly conserved portion of the MMTV-env gene with only $16 \%$ homology to the prototype HERV-K10 human endogenous retrovirus, Wang et al. ${ }^{8}$ from Beatriz Pogo's lab, were able to demonstrate MMTV- env specific sequences in $38.5 \%$ of the 314 breast carcinomas and in $6.9 \%$ of the 29 breast fibroadenoma samples, compared to only $1.8 \%$ of 107 samples of normal breast reduction mammoplasty tissues. $^{8}$ The specificity of these results was confirmed by hybridization to an MMTV-env specific probe, as well as by sequencing of the PCR product from 15 of the positive tumours. In all cases the homology to the MMTV-env gene ranged from 95 to $99 \%$, while homology to the HERV-K10-env gene was found to be less than $15 \%$ and to other viral and human genes was found to be maximally $18 \%{ }^{9}$

The Wang study catalysed a whole series of similar studies using PCR primers and nested primers in an attempt to correlate the presence of the indicative MMTV specific $660 \mathrm{bp}$ env sequence with mammary tumorigenesis. ${ }^{8}$ We analysed 20 studies reported in 17 publications in which PCR was used either on total DNA extracted from tumour and normal tissues. Of 15 pure PCR studies, in which tissues from patients with breast cancer were compared to normal non-tumour tissue using primers located within or immediately adjacent to the $660 \mathrm{bp}$ MMTV specific region identified by Wang et al., 11 studies showed a positive correlation between the presence of the indicative MMTV signal and breast tumour tissue at the $p<0.01$ significance level, two studies, from Morocco and Saudi Arabia, showed the correlation at the $p<$ 0.05 significance level, and two studies from Italy and Mexico, showed no significant correlation (Table 1). Based on a metaanalysis of PCR based case control studies by Wang et al. plus later studies, the overall prevalence of MMTV-like gene sequences is 15fold higher in human breast cancer than in normal human breast tissue controls. ${ }^{10}$ This is shown in Fig. 1. The prevalence of MMTVlike sequences in breast cancers varies from a high of $75 \%$ in Tunisia to a low of $6 \%$ in Saudi Arabia. The prevalence in normal control human breast tissues varies from $33 \%$ in Morocco to zero

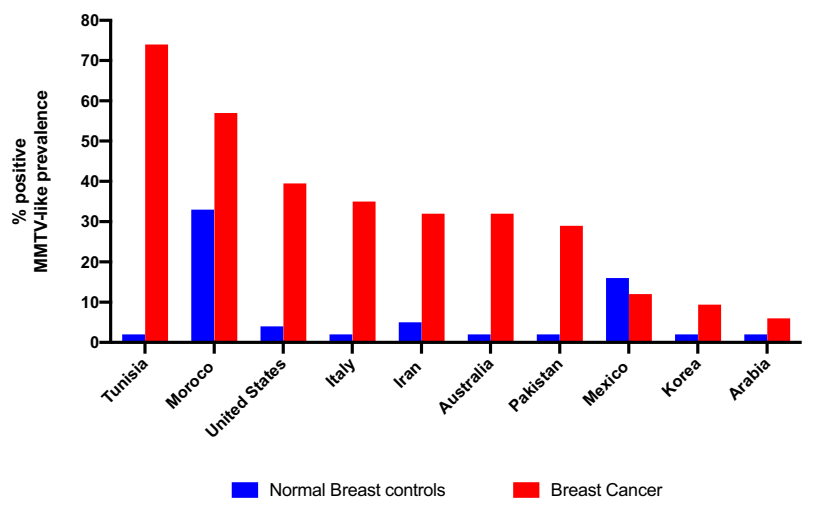

Fig. 2 Prevalence of MMTV-like gene sequences in invasive human breast cancers compared to normal human breast tissues in women from 10 selected countries (based on Table 1). The prevalence of MMTV-like sequences in breast cancers varies from a high of $75 \%$ in Tunisia to a low of $6 \%$ in Saudi Arabia. The prevalence in normal control human breast tissues varies from 33\% in Morocco to zero in, Tunisia, Italy, Australia, Pakistan, Korea and Saudi Arabia

in, Tunisia, Italy, Australia, Pakistan, Korea and Saudi Arabia. This is shown in Fig. 2.

\section{Laser microdissection}

To further investigate the presence of a signal from a $200 \mathrm{bp}$ subfragment of the highly conserved MMTV env region laser microdissection techniques were used to study breast cancer epithelial cells followed by real-time PCR analysis. ${ }^{11}$ MMTV was identified in $40(82 \%)$ of 49 ductal carcinoma in situ specimens and 7 (35\%) of 20 invasive ductal carcinoma specimens compared to no identification in 20 normal breast specimens from reduction mammoplasty.

While the outcome of studies of MMTV in human breast cancer are generally consistent, several research groups have experienced difficulties when using PCR techniques for the identification of MMTV gene sequences. This is illustrated by the experience of Park et al. who could not identify MMTV gene sequences in breast cancers in Australian women despite their previous identification by Ford et al. ${ }^{12,13}$ Pogo et al. demonstrated by laboratory experiments that the methods used by Park et al. were inadequate. $^{14}$

\section{CONTAMINATION}

Perzova et al. have challenged the validity of the identification of MMTV gene sequences in human breast cancers. ${ }^{15}$ They argue that many of the findings outlined above and which are based on PCR technology, are due to contamination by MMTV with mice and other rodents as the original source. There is laboratory based evidence, which demonstrates that this view is incorrect. Potentially contaminating rodent DNA can be identified by testing PCR products for the presence of murine mitochondrial genomic DNA plus the detection of murine intracisternal A particle long terminal repeats. These techniques have been used to exclude contamination by rodent DNA in five independent laboratories. ${ }^{16-20}$

\section{Whole-genome sequencing}

There are difficulties with the use of whole-genome sequencing for the identification of MMTV in human breast cancer. MMTV sequences were identified in only three of over 800 breast cancer specimens from The Cancer Genome Atlas (TCGA) as compared to the frequent identification by PCR techniques of MMTV sequences in over $40 \%$ of breast cancers. ${ }^{21}$ This low identification of MMTV by whole-genome sequencing is possibly due to its limited 
integration into the host human genome. There are similar experiences with respect to other retroviruses, including bovine leukemia virus and human immunodeficiency virus.

Lehrer and Rheinstein have noted that MMTV pol, ENV and other genes are present in breast tumours and normal breast tissues. ${ }^{22}$ They did not identify the MMTV env gene in the human genome or the genomes of other primates. They suggest this finding indicates that the MMTV ENV sequences that were identified in breast tumours and normal breast tissues were from an infection.

\section{Serology}

The prevalence of MMTV antibodies in the serum of women with breast cancer is consistently five-fold higher than in the sera of women with benign breast conditions or in normal women (Table 2). Different methods have been used to measure the specificity of the antibodies in these studies. Regardless of the methods the outcomes are similar with the exception of Kovarik and Goedert et al. ${ }^{23,24}$ Kovarik et al. ${ }^{23}$ identified MMTV antibodies in 2 (3\%) of 60 serum samples from women with breast cancer compared to 0 $(0 \%)$ of normal controls. ${ }^{23}$ Goerdert et al. did not identify any MMTV antibodies in the sera of 92 women with breast cancer. ${ }^{24}$ The outcomes of the Goedert et al. study may not be valid because the sera used was collected 10-15 years before the study and frozen several times. Repeated thawing and freezing degrades antibody proteins. ${ }^{25}$ In addition MMTV gp52 was not identified in either the positive controls or the sera from the patients with breast cancer in the Goedert et al. study.

Laboratory workers exposed to MMTV have showed serological responses to the MMTV env (gp52 and gp34), and gag (p12, p18, p28) proteins. $^{26}$

\section{EPIDEMIOLOGY}

MMTV-like sequences have been identified in 30 and $40 \%$ of invasive breast cancers in Western countries and between 10 and $20 \%$ in Asian countries. The differences may be due to the geographical differences in the prevalence of MMTV in mice-, which may influence the prevalence of MMTV in humans. ${ }^{27}$ It has been proposed that the higher the prevalence of one species of house mouse, Mus domesticus, the higher the prevalence of human breast cancer. ${ }^{27}$

There have not been any studies of associations between MMTV-like positive breast cancers and immunophenotypes. While there are correlations between MMTV-like positive human invasive breast cancers and histological characteristics similar to MMTVpositive mouse mammary tumors, there do not appear to be any associations with histological types. ${ }^{21}$

Because breast cancer is more prevalent among women of higher socio-economic status, Lehrer and Rheinstein have hypothesised this may be due to exposure to infection by MMTV from mice during early life among girls of low socio-economic status and hence gaining early immunity. ${ }^{28}$

\section{MMTV PREVALENCE IN BRCA HEREDITARY COMPARED TO SPORADIC BREAST CANCERS}

In an intriguing new study, Naccarato et al. report the identification of MMTV-like gene sequences (MMTV) in 17 (30.3\%) of 56 sporadic human breast cancers as compared to $2(4.2 \%)$ of 47 hereditary human breast cancers. ${ }^{20}$ The difference is statistically significant $(p=0.001)$. As hereditary breast cancers are known to be associated with BRCA gene mutations and are not associated with oncogenic viruses, the implication of these observations is that MMTV has a likely role in sporadic breast cancers.

\section{TRANSFORMATION}

Proteins expressed by the MMTV envelope gene are capable of malignantly transforming normal human breast epithelial cells. ${ }^{29}$ This shown in Fig. 3. ${ }^{29}$ MMTV envelope protein p14 overexpression can function in an oncogenic capacity. ${ }^{30}$ MMTV encoded proteins (such as Rem, Sag, Naf) or as yet uncharacterised proteins analogous to those of other complex retroviruses such as Tax may also have a role in breast cancer.

\section{SPECIFICITY}

MMTV-like virus is not specific for breast cancer. MMTV-like virus has been identified in a number of human cancers including breast, lymphoma, prostate, liver and endometrial liver, endometrial, ovary, prostate skin and lung cancers and also in biliary cirrhosis. ${ }^{31,32}$ Apart from breast cancer, there is no evidence available to determine whether MMTV is causal in these cancers. MMTV in mice is also associated with mammary, lymphomas, salivary gland tumours and with renal adenocarcinoma. ${ }^{33}$

\section{TEMPORALITY}

MMTV infection and subsequent breast cancer time sequence MMTV has been identified by both PCR and immunohistochemistry techniques in normal and benign human breast tissues before the development of MMTV-positive breast cancer 1-11 years later in the same women. ${ }^{17,34}$

\section{BIOLOGICAL GRADIENT}

As human breast cancer progresses, the MMTV viral load increases but it falls in late stage invasive breast cancer. ${ }^{11,12}$ The lessened identification of MMTV in advanced breast cancer may be due to the breakdown of cell physiology.

\section{PLAUSIBILITY - IDENTIFICATION OF MMTV IN OTHER MAMMALS}

MMTV is the proven cause of mammary cancers in wild (feral) mice. ${ }^{33}$ Gene sequences with high homology to MMTV have been identified in rhesus monkeys and cats although the tissues studied were not mammary tumours. ${ }^{35}$ MMTV-like gene sequences have been identified in $20 \%$ of mammary tumours in dogs and $33 \%$ of mammary tumours in cats. ${ }^{36}$ Civita et al. ${ }^{19}$ have identified MMTV gene sequences in cat mammary tumours but not in dog mammary tumours. ${ }^{19}$ The findings by Civita et al., based on PCR, were confirmed by immunohistochemistry using MMTV p14 antibodies. ${ }^{19}$ Accordingly, it is plausible that MMTV may also cause mammary tumours in humans.

\section{EXPERIMENTAL EVIDENCE}

Capacity of MMTV to infect human breast epithelial cells MMTVs can infect human breast cells in culture. ${ }^{37,38}$ This shown in Fig. 4. Faschinger et al. demonstrated that when MMTVs infect human mammary epithelial cells they randomly integrate their genomic information into the human genome of the infected cell. ${ }^{38}$ Following integration of MMTV into the human genome of the infected mammary epithelial cells the flanking sequences are of human and not mouse origin. This is an indication of an exogenous infection rather than contamination. ${ }^{39}$ Integration of MMTV into the human genome appears to be random and in multiple locations. Faschinger et al. also demonstrated there is a similar pattern of random MMTV integration into the mouse genome. $^{39}$

Overall, this evidence indicates an infective transmission of MMTV in humans. 


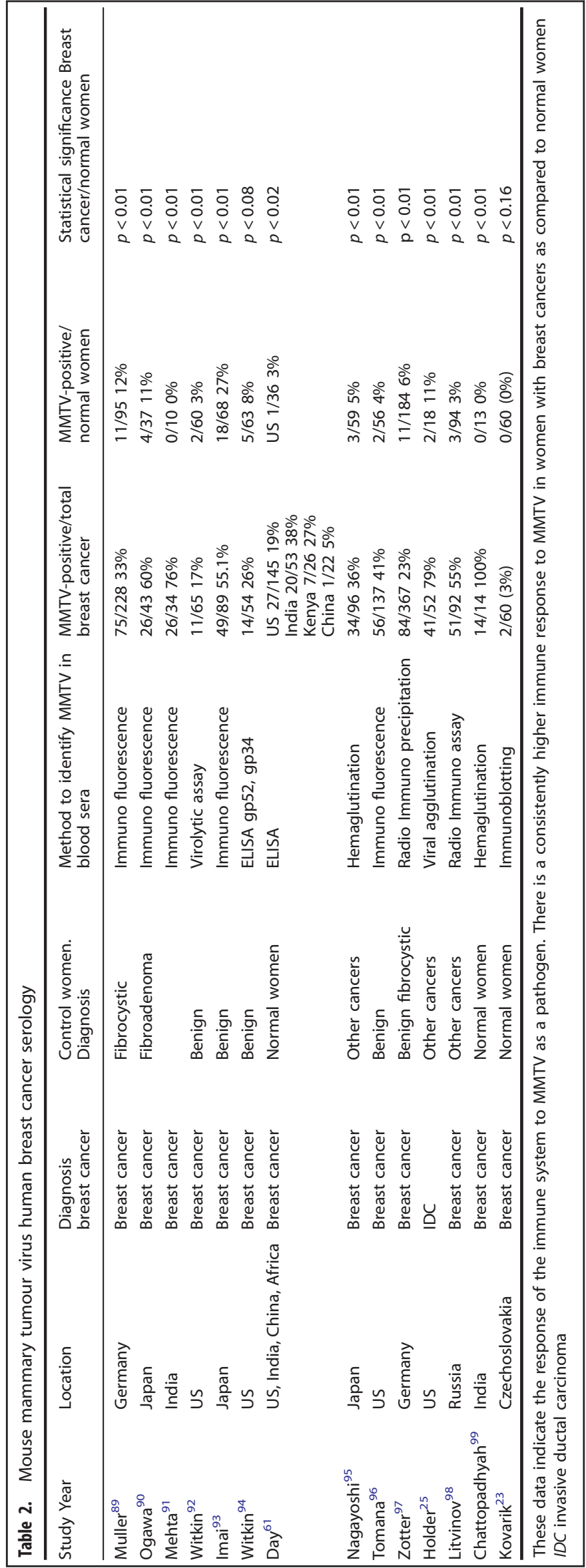

\section{ANALOGY}

Analogy between MMTV-associated mouse mammary tumours and MMTV-associated human breast cancer

The biology of MMTV in mice is very similar to the apparent biology of MMTV in humans. ${ }^{33}$ In mice MMTV is spread by the milk of infected mouse mothers and is acquired by suckling pups. MMTV is also present in the salivary glands of mice. ${ }^{33}$ Ingested MMTV enters T and B cells located in Peyer's patches of the gastrointestinal tract of infected mouse pups. This spread of MMTV infection requires activation of $T$ and $B$ lymphocytes by the viral superantigen (SAg). SAg activation allows the virus to amplify in lymphocytes prior to transmission of the virus to mammary epithelial cells. Ultimately the virus is transported by infected lymphocytes to the mammary glands where oncogenic transformation takes place. In humans, MMTV is present in saliva and human milk and after ingestion infects $T$ and $B$ lymphocytes located in the Peyers patches in the intestine, which are activated by SAg and randomly infects and integrates into the human genome located in normal breast epithelial cells. ${ }^{18,40-42}$ The possible MMTV etiopathogenesis in human breast cancer is shown in Fig. 5.

The evidence related to MMTV-associated human breast cancer and MMTV-associated mouse mammary tumours, is remarkably similar.

(1) MMTV has been identified globally in human breast cancer and mouse mammary tumours. ${ }^{10,27}$ The highest incidence of breast cancer occurs in locations where Mus domesticus is the resident native or introduced species of house mouse. ${ }^{27}$ MMTV prevalence is higher in Mus domesticus mice than in other mice strains.

(2) Virus particles have been identified in human milk from women with breast cancers. ${ }^{43}$ These particles in human milk are physically similar to MMTV particles identified in mice. ${ }^{43}$ Virus particles from human breast cancer cells contain MMTV sequences. ${ }^{9}$ MMTV gene sequences have been identified in human milk from normal lactating Australian and US women. ${ }^{40,41}$

(3) The nucleotide sequences and structure of MMTV-like viral sequences that have been identified in human breast cancer tissues are virtually identical to MMTV sequences identified in mouse mammary tumours. ${ }^{9,44}$

(4) Abnormal cancer-related gene expression is similar in mouse mammary tumours and human breast cancers. ${ }^{45,46}$ Klein et al. identified the same 63 genes, which were differentially expressed in over $75 \%$ of both human breast cancers and mouse mammary tumours. ${ }^{46}$

(5) MMTV-associated envelope and capsid protein expression is similar in both mouse mammary tumours and human breast cancers. $^{30,47,48}$

(6) Superantigen (SAg) expression. MMTV SAgs play an essential role in MMTV-associated mouse mammary tumours. ${ }^{49}$ MMTV-infected B lymphocytes and dendritic cells lead to the expression of virus SAgs, which in turn stimulate T-cells to produce cytokines that encourage the proliferation of infected B lymphocytes thereby forming a reservoir of infected cells. MMTV is then conducted throughout the body by these lymphocytes, which facilitate the entry of MMTV into its target organ (the mammary glands or other organs). MMTV SAg is highly expressed in MMTV-associated human breast cancer and may have a similar role in humans as it does in the mouse. Human T cells respond to MMTV SAg. ${ }^{50,51}$

(7) MMTV infects intestinal T and B lymphocytes and randomly integrates into both the mouse and human genome. ${ }^{33,38,39}$ Similarly, MMTV-infected human lymphocytes can be found in women with MMTV-positive breast cancer. ${ }^{52-54}$ In immunocompromised mice injected with wild-type MMTV, 
A

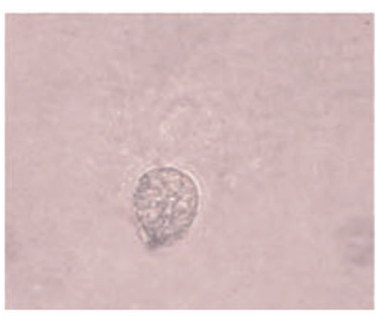

B

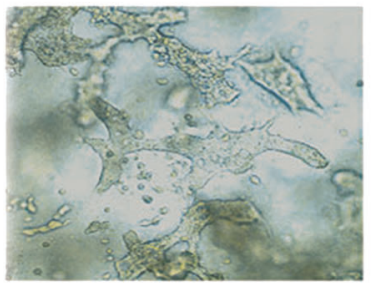

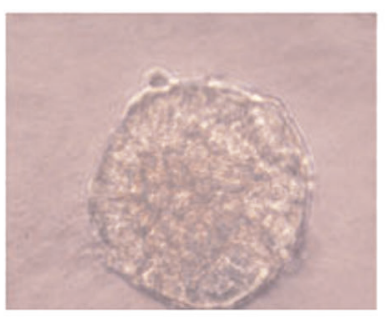
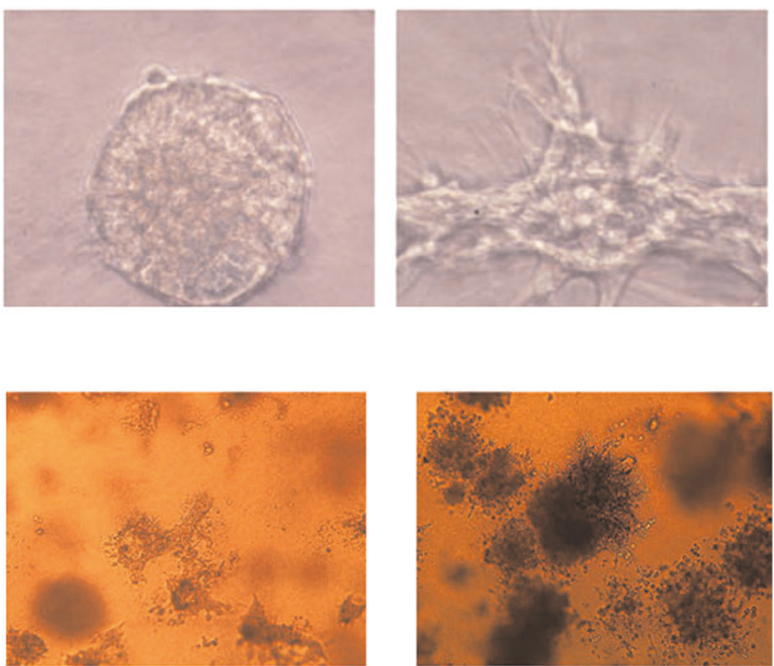

Fig. 3 Mouse mammary tumour virus envelope proteins transform normal human mammary epithelial cells into invasive breast cancer cells. The images demonstrate transformation from a normal mouse mammary cells to invasive cancer cells $\mathbf{b}$ normal human breast ductal cells following experimental exposure to MMTV. Figure used with permission from Rockefeller University Press under licence number 4672720172161 . The Figure is based on two separate figures in Katz et al. ${ }^{29}$

non-infected
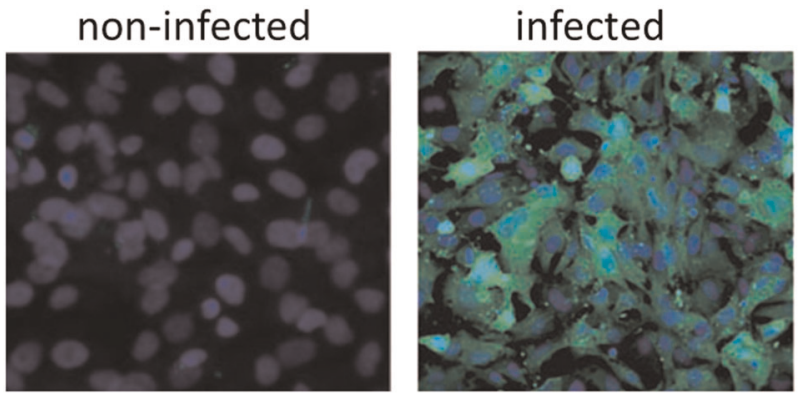

Fig. 4 Mouse mammary tumour virus infects human breast epithelial cells. The detection of MMTV in the cells is by MMTVenhanced green fluorescent protein (based on experiments conducted by Konstantoulas and Indik ${ }^{38}$ )

there is no infection of peripheral lymphocytes or virus spread to the mammary glands. ${ }^{50}$ The risk of breast cancer is reduced by $44 \%$ in immunocompromised human patients. ${ }^{55}$ This is in contrast to immunocompromised patients with other cancers whose risk of cervical and other cancers increases markedly. ${ }^{56}$ These observations are compatible with the notion that an intact immune system is required for the transmission of MMTV by lymphocytes in both mice and humans.

(8) MMTV-associated tumour histology appears to be similar in both mouse mammary tumours and MMTV-positive human breast cancers. $^{21,57}$ It is of interest that this similarity mainly applies to mouse mammary tumours in mice who were infected with MMTV during the neonatal period (for more detail see the section on transmission below ${ }^{58}$ ). However, the MMTV-associated histology is not necessarily specific to human breast cancer as there are similarities to other cancers such as squamous cell skin cancers. A recent study has shed some clarity to this issue. Based on a study of genes and histology, Hollern et al. have shown that many features of murine tumour histologies are conserved in both mouse and humans and in several different cancer types. ${ }^{59}$

(9) Antibodies to the MMTV surface envelope gp52 are present in the serum and mammary glands of feral mice. ${ }^{60}$ Antibodies to MMTV gp52 have been identified in the serum of $2.8 \%$ of normal US women. ${ }^{61}$ However, the repeated identification of MMTV gp52 antibody in human breast cancers has yet to be confirmed by modern methods in humans. ${ }^{24}$

(10) Hormones. Mammary tumours in some strains of mice are hormone dependent. $^{62}$ In humans, MMTV hormonal response elements in breast cancers appear to promote cell growth as they do in mice. ${ }^{63}$

(11) Wnt-1 is an oncogene, which is highly expressed in MMTVassociated mouse mammary tumours. ${ }^{33}$ In humans, there is suggestive evidence that the influence of MMTV on human breast tissues cells leads to abnormally high Wnt-1 expression. ${ }^{64}$

MMTV has been identified in mammary tissues in feral mice and in normal human breast tissues prior to the development of MMTV-positive mouse mammary tumours and MMTV-positive human breast cancers. ${ }^{17,34}$

\section{MMTV TRANSMISSION}

The most plausible means of transmission of MMTV in humans is via human saliva. ${ }^{18}$ MMTV gene sequences are present in saliva in $27 \%$ of normal children, $11 \%$ of normal adults and $57 \%$ of women with breast cancer. ${ }^{18}$ MMTV-like gene sequences have been identified in human parotid glands-the source of saliva. ${ }^{18}$ MMTV can infect adult mice via nasal lymphoid tissue. Humans have well developed lymphatic structures in the mouth and nose (tonsils and adenoids), which are possible entry points for MMTV.

The father, mother and daughter of a family living together, all developed MMTV-associated breast cancer. ${ }^{65}$ The MMTV env gene sequences identified in each family member was at least $98 \%$ homologous to the MMTV env sequences found in laboratory mouse strains. This observation supports the evidence that transmission of MMTV is likely to be by saliva.

The prevalence of MMTV in human milk is significantly higher among women who are at greater than normal risk of breast cancer. ${ }^{41}$ This suggests that human milk is a possible means of MMTV transmission.

MMTV gene sequences have been identified in mammary tumours in dogs and cats. ${ }^{19,35,36}$ Women with companion dogs are 
Human to human spread of MMTV via saliva

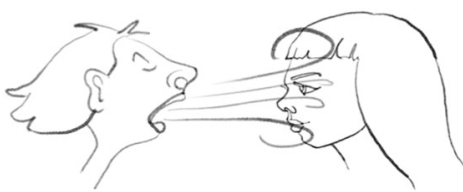

MMTV infects B and T lymph cells in Peyer's Patches located in small intestine

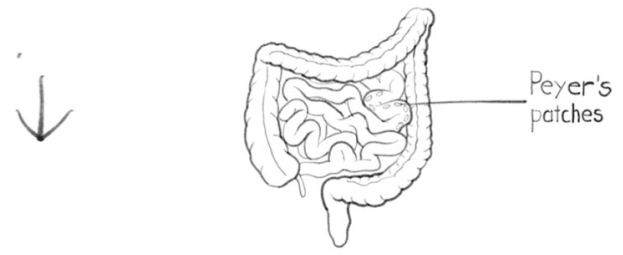

MMTV virus encoded superantigen activates circulating B and T lymph cells
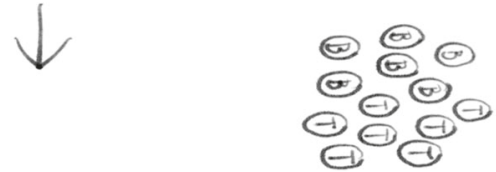

Transmission of MMTV to breast via B and T lymph cells. Random integration of MMTV into human genome in breast epithelial cells. Transformation.

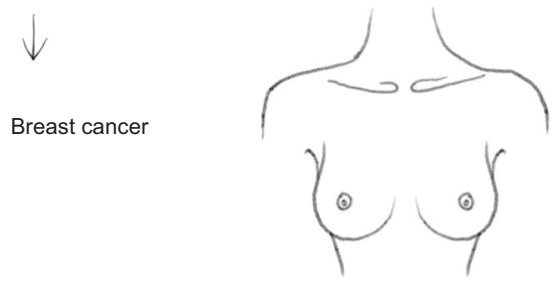

Fig. 5 Possible etiopathogenesis of MMTV-associated human breast cancer. The evidence in support of this possible etiopathogenesis for MMTV in human breast cancer is not as robust as that for MMTV in mice but is substantial. ${ }^{18,29,37,38,42,51}$

at twice the expected risk of breast cancer, which suggests MMTV could be transmitted in dog saliva to humans. ${ }^{66}$

In many countries it is permissible for $1 \%$ in weight of cereals to consist of mouse or rat faecal material. ${ }^{67}$ Because MMTV is endemic in many rodent populations, transmission by consumption of uncooked cereals and other foods is possible.

Overall this evidence suggests that while human to human saliva is the most likely means of MMTV-like transmission. Additional means of transmission are also possible.

\section{MMTV ONCOGENIC MECHANISMS IN HUMAN BREAST CANCER}

The underlying causal mechanisms by which MMTV may cause human breast cancer are far from clear. With respect to laboratory mice it has been demonstrated that integration of MMTV proviral DNA into the mouse genome near one or more of the protooncogenes such as Wnt-1 and Fgf, is associated with the development of mouse mammary tumours. However, more recent studies have indicated that MMTV oncogenesis in mouse mammary tumours is more complex than insertional oncogenesis. MMTV envelope genes encode immunoreceptor tyrosine-based activation motif (ITAM) containing proteins, which are capable of malignantly transforming mouse mammary epithelial cells. ${ }^{29,47}$

The relatively low levels of MMTV-like gene sequences that are detected in human breast cancers suggest that the virus is affecting oncogenesis by mechanisms in addition to MMTV insertion. Additional mechanisms by which MMTVs can contribute to human breast oncogenesis include (i) proteins expressed by the MMTV envelope gene and which are capable of malignantly transforming normal human breast epithelial cells, ${ }^{29}$ (ii) MMTV envelope protein p14 overexpression can function in an oncogenic capacity $^{30}$ (iii) MMTV encoded proteins (such as Rem, Sag, Naf) or as yet uncharacterised proteins analogous to those of other complex retroviruses such as Tax may also have a role in breast cancer. The envelope proteins of Jaagsiekte Sheep Retrovirus (JSRV) which like MMTV is a beta retrovirus, can directly transform cells and offers a precedent for the ability of viral envelope proteins to malignantly transform cells. ${ }^{68}$ There is also the intriguing possibility that MMTV and human endogenous retroviruses may interact and thus also play a role. ${ }^{30}$

The enzyme $A P O B E C 3 B$ is an additional potential oncogenic mechanism. APOBEC3B is an enzyme, which inhibits retrovirus replication. In mice, APOBEC3 has been shown to inhibit MMTV infections and viral replication and to inhibit milk borne MMTV virions. $^{69}$ In humans, inactivating mutations and deletions in APOBEC3B appear to play a role in breast cancer development. ${ }^{70} \mathrm{~A}$ deletion polymorphism in the APOBEC3B gene cluster on chromosome 22 is associated with elevated breast cancer risk and a specific APOBEC mutation pattern has been described in a number of cancers, including breast cancer. ${ }^{70}$ This mutation pattern is linked to APOBEC3B expression specifically in breast cancer and leads to DNA damage that could select TP53 inactivation, thereby resulting in tumour heterogeneity. ${ }^{69}$ Human papilloma viruses have been shown to alter the expression of $A P O B E C 3 B$, which may reduce its protective effects against MMTV. ${ }^{71,72}$ It has also been shown that there is a significant increase in APOBEC-mediated mutagenesis in HER+/HER2 metastatic breast tumours as compared to early stage primary breast cancer. Taken together, these studies suggest that the APOBEC family and particularly APOBEC $3 \mathrm{~B}$, may play a key role in the early stages of breast cancer induction.

\section{MULTIPLE ONCOGENIC VIRUSES AND BREAST CANCER}

There is substantial evidence that additional oncogenic viruses may have roles in breast cancer. These are bovine leukemia virus, high risk human papilloma viruses and Epstein Barr virus (EBV). ${ }^{73}$ As discussed above the role of these viruses is complex and may be indirect. This is the same way that human immunodeficiency virus (HIV) has an indirect role in Kaposi's sarcoma. ${ }^{73}$ In addition, there may be collaboration between oncoviruses in breast cancer. For example there is evidence that EBV and human papilloma viruses may interact and lead to increased cell proliferation. ${ }^{73,74}$

\section{PREVENTION}

Three very different methods using experimental mice, have been used to develop protection against MMTV induced mouse mammary tumours. The first method has been the use of formalin inactivated mouse mammary tumour viruses as vaccines. The second method has been the use of MMTV based peptides and proteins. The third method is the use of vaccines prepared from cells of mouse mammary tumours. Each of these methods has been successful in reducing either MMTV infections in mice or mouse mammary tumour development. Recently the Hochman group working in Israel, together with the Bevilacqua group in Italy, have identified specific MMTV envelope proteins, which can potentially be used for both prevention and treatment of MMTVassociated human breast and other cancers. ${ }^{75}$

\section{CONCLUSIONS}

The evidence is sufficiently detailed and comprehensive to conclude: 
(i) DNA gene sequences, which are almost identical to mouse mammary tumour virus (MMTV) sequences are present in up to $40 \%$ of human breast cancers.

(ii) MMTV-like gene sequences are up to 15-fold more prevalent in human breast cancers as compared to normal and benign breast tissues in the same populations of women.

(iii) MMTV-like gene sequences and MMTV p14 proteins can be identified in normal and benign breast tissues 1-11 years before the development of MMTV-like positive breast cancer in the same women.

(iv) MMTV can infect human mammary epithelial cells and integrate at random into the human genome located in those cells.

(v) The prevalence of MMTV antibodies in sera from women with breast cancer is five-fold higher than in normal women.

(vi) Proteins expressed by the MMTV envelope gene can malignantly transform normal human breast epithelial cells.

(vii) Saliva has been identified as the most plausible means of transmission from human to human and possibly from dogs to humans.

(viii) MMTV-like gene sequences are present in human milk from normal lactating women and with increased prevalence in milk from women at risk of breast cancer.

(ix) MMTV-like gene sequences have also been identified in dogs, cats, monkeys and other mammals including mice and rats.

(x) There is a striking similarity in the features of MMTV-like virus associated human breast cancer compared to MMTVassociated mouse mammary tumours. The nucleotide sequences and structure of the MMTV-like and MMTV viruses are almost identical. Abnormal gene expression, possibly histology, superantigen expression, MMTV infection of $\mathrm{B}$ and $\mathrm{T}$ lymphocytes and hormone dependence are all similar in both MMTV-like positive human breast cancers and MMTV-associated mouse mammary tumours.

The evidence with respect to MMTV being responsible for a subset of approximately $30-40 \%$ of human breast cancers in Western countries and $10-20 \%$ in Asian countries is compelling. An exception is the lack of detailed information about causal mechanisms.

A role for MMTV-like viruses in human breast cancer is highly likely.

Received: 31 March 2019; Accepted: 15 October 2019; Published online: 07 November 2019

\section{REFERENCES}

1. Hill, A. B. The environment and disease: association or causation? Proc. R. Soc. Med. 58, 295-330 (1965)

2. Bittner, J. J. Some possible effects of nursing on the mammary gland tumor incidence in mice. Science 84, 162 (1936).

3. Graff, S., Moore, D. H., Stanley, W. M., Randall, H. T. \& Haagensen, C. D. Isolation of mouse mammary carcinoma virus. Cancer 2, 755-762 (1949).

4. Duesberg, P. H. \& Blair, P. B. Isolation of the nucleic acid of mouse mammary tumor virus (MMTV). Proc. Natl Acad. Sci. USA 55, 1490-1497 (1966).

5. Callahan, R., Drohan, W., Tronick, S. \& Schlom, J. Detection and cloning of human DNA sequences related to the mouse mammary tumor virus genome. Proc. Natl Acad. Sci. USA 79, 5503-5507 (1982).

6. Szakacs, J. G. \& Moscinski, L. C. Sequence homology of deoxyribonucleic acid to mouse mammary tumor virus genome in human breast tumors. Ann. Clin. Lab Sci. 21, 402-412 (1991).

7. Ono, M., Yasunaga, T., Miyata, T. \& Ushikubo, H. Nucleotide sequence of human endogenous retrovirus genome related to the mouse mammary tumor virus genome. CANJ Virol. 60, 589-598 (1986).

8. Wang, Y. et al. Detection of mammary tumor virus env gene-like sequences in human breast cancer. Cancer Res. 55, 5173-5179 (1995).

9. Melana, S. M. et al. Characterization of viral particles isolated from primary cultures of human breast cancer cells. Cancer Res. 67, 8960-8965 (2007).
10. Wang, F. et al. Mouse mammary tumor virus-like virus infection and the risk of human breast cancer: a meta-analysis. Am. J. Transl. Res. 6, 248-266 (2014).

11. Mazzanti, C. M. et al. A mouse mammary tumor virus env-like exogenous sequence is strictly related to progression of human sporadic breast carcinoma. Am. J. Pathol. 179, 2083-2090 (2011).

12. Ford, C. E., Tran, D. D., Deng, Y. M., Rawlinson, W. D. \& Lawson, J. S. Mouse mammary tumour like virus prevalence in breast tumours of Australian and Vietnamese women. Clin. Cancer Res. 9, 1118-1120 (2003).

13. Park, D. J., Southey, M. C., Giles, G. G. \& Hopper, J. L. No evidence of MMTV-like env sequences in specimens from the Australian Breast Cancer Family Study. Breast Cancer Res. Treat. 125, 229-235 (2011).

14. Pogo, B. G., Melana, S. M., Moran, H. \& Holland, J. F. Presence of MMTV-like env gene sequences in human breast cancer. Breast Cancer Res. Treat. 125, 295-297 (2011).

15. Perzova, R. et al. Is MMTV associated with human breast cancer? Maybe, but probably not. Virol. J. 14, 196 (2017).

16. Cedro-Tanda, A. et al. Prevalence of HMTV in breast carcinomas and unaffected tissue from Mexican women. BMC Cancer 14, 942 (2014).

17. Nartey, T. et al. Mouse mammary tumor-like virus (MMTV) is present in human breast tissue before development of virally associated breast cancer. Infect. Agent Cancer 12, 1 (2017).

18. Mazzanti, C. M. et al. Human saliva as route of inter-human infection for mouse mammary tumor virus. Oncotarget 6, 18355-18363 (2015).

19. Civita, P. et al. Mouse mammary tumour virus-like env nucleotide and p14 signal peptide are present in feline mammary carcinomas, but not in neoplastic or dysplastic canine mammary lesions. PLOS ONE 13, e0200839 (2018).

20. Naccarato, A. G. et al. MMTV-like human exogenous betaretrovirus sequences are associated to sporadic but not to hereditary human breast carcinoma. Aging 11, 7236-7241 (2019).

21. Lawson, J. S. et al. Association of mouse mammary tumour virus and human breast cancer: histology, immunohistochemistry and PCR analyses. Front. Oncol 8, 141 (2018).

22. Lehrer, S. \& Rheinstein, P. H. Mouse mammary tumor viral env sequences are not present in the human genome but are present in breast tumors and normal breast tissues. Virus Res. 266, 43-47 (2019b).

23. Kovarik, A., Hlubinova, K., Prachar, J., Simkovic, D. \& Knotek, J. No significant correlation between specific antibodies to mouse mammary tumour virus and human cancer. Br. J. Cancer 60, 572-575 (1989).

24. Goedert, J. J., Rabkin, C. S. \& Ross, S. R. Prevalence of serologic reactivity against four strains of mouse mammary tumour virus among US women with breast cancer. Br. J. Cancer 94, 548-551 (2006).

25. Holder, W. D. Jr. \& Wells, S. A. Jr. Antibody reacting with the murine mammary tumor virus in the serum of patients with breast carcinoma: a possible serological detection method for breast carcinoma. Cancer Res. 43, 239-244 (1983).

26. Dion, A. S., Girardi, A. J., Williams, C. C. \& Pomenti, A. A. Serologic responses to murine mammary tumor virus (MuMTV) in MuMTV-exposed laboratory personnel. J. Natl. Cancer Inst. 76, 611-619 (1986).

27. Stewart, T. H. M., Sage, R. D., Stewart, A. F. R. \& Cameron, D. W. Breast cancer incidence highest in the range of one species of house mouse, Mus domesticus. Br. J. Cancer 82, 446-451 (2000).

28. Lehrer, S. \& Rheinstein, P. H. The virology of breast cancer: viruses as the potential causative agents of breast tumorigenesis. Disco. Med. 27, 163-166 (2019b).

29. Katz, E. et al. MMTV Env encodes an ITAM responsible for transformation of mammary epithelial cells in three-dimensional culture. J. Exp. Med. 201, 431-439 (2005).

30. Feldman, D. et al. The signal peptide of mouse mammary tumor virus-env: a phosphoprotein tumor modulator. Mol. Cancer Res. 10, 1077-1086 (2012).

31. Johal, $\mathrm{H}$. et al. DNA of mouse mammary tumor virus-like virus is present in human tumors influenced by hormones. J. Med. Virol. 82, 1044-1050 (2010).

32. Deligdisch, L. et al. Human mammary tumor virus (HMTV) in endometrial carcinoma. Int J. Gynecol. Cancer 23, 1423-1428 (2013).

33. Dudley, J. P., Golovkina, T. V. \& Ross, S. R. Lessons learned from mouse mammary tumor virus in animal models. ILAR J. 57, 12-23 (2016).

34. Lawson, J. S. \& Glenn, W. K. Multiple oncogenic viruses are present in human breast tissues before development of virus associated breast cancer. Infect. Agent Cancer 16, 55 (2017).

35. Szabo, S., Haislip, A. M. \& Garry, R. F. Of mice, cats, and men: is human breast cancer a zoonosis? Microsc Res. Tech. 68, 197-208 (2005).

36. Hsu, W. L. et al. Mouse mammary tumor virus-like nucleotide sequences in canine and feline mammary tumors. J. Clin. Microbiol. 48, 4354-4362 (2010).

37. Indik, S., Günzburg, W. H., Kulich, P., Salmons, B. \& Rouault, F. Rapid spread of mouse mammary tumor virus in cultured human breast cells. Retrovirology 4, 73 (2007).

38. Konstantoulas, C. J. \& Indik, S. C3H strain of mouse mammary tumour virus, like GR strain, infects human mammary epithelial cells, albeit less efficiently than murine mammary epithelial cells. J. Gen. Virol. 96, 650-662 (2015). 
39. Faschinger, A. et al. Mouse mammary tumor virus integration site selection in human and mouse genomes. J. Virol. 82, 13 (2008).

40. Johal, H. et al. Mouse mammary tumor like virus sequences in breast milk from healthy lactating women. Breast Cancer Res. Treat. 129, 149-155 (2011)

41. Nartey, T. et al. Human Mammary Tumor Virus (HMTV) sequences in human milk. Infect. Agent Cancer 9, 20 (2014).

42. Lushnikova, A. A., Kryukova, I. N., Rotin, D. L. \& Lubchenko, L. N. Detection of the env MMTV-homologous sequences in mammary carcinoma patient intestine lymphoid tissue. Dokl. Biol. Sci. 399, 423-426 (2004).

43. Moore, D. H. et al. Search for a human breast cancer virus. Nature 229, 611-614 (1971).

44. Nishio, M. et al. Complete nucleotide sequence of mouse mammary tumor virus from JYG Chinese wild mice: absence of bacterial insertion sequences in the cloned viral gag gene. Breast Cancer 1, 89-94 (1994).

45. Callahan, R. et al. Genes affected by mouse mammary tumor virus (MMTV) proviral insertions in mouse mammary tumors are deregulated or mutated in primary human mammary tumors. Oncotarget 3, 1320-1334 (2012).

46. Klein, A. et al. Comparison of gene expression data from human and mouse breast cancers: identification of a conserved breast tumor gene set. Int J. Cancer 121, 683-688 (2007).

47. Ross, S. R. MMTV infectious cycle and the contribution of virus-encoded proteins to transformation of mammary tissue. J. Mammary Gland Biol. Neoplasia. 13, 299-307 (2008).

48. Melana, S. M. et al. Detection of human mammary tumor virus proteins in human breast cancer cells. J. Virol. Methods 163, 157-161 (2010).

49. Wei, W. Z., Gill, R. F. \& Wang, H. Mouse mammary tumor virus associated antigens and superantigens-immuno-molecular correlates of neoplastic progression. Semin Cancer Biol. 4, 205-213 (1993).

50. Labrecque, N., McGrath, H., Subramanyam, M., Huber, B. T. \& Sékaly, R. P. Human $\mathrm{T}$ cells respond to mouse mammary tumor virus-encoded superantigen: $\mathrm{V}$ beta restriction and conserved evolutionary features. J. Exp. Med. 177, 1735-1743 (1993)

51. Wang., Y. et al. A mouse mammary tumor virus-like long terminal repeat superantigen in human breast cancer. Cancer Res. 64, 4105-4111 (2004).

52. Crépin, M. et al. Sequences related to mouse mammary tumor virus genome in tumor cells and lymphocytes from patients with breast cancer. Biochem Biophys. Res. Commun. 118, 324-331 (1981).

53. Smorodinsky, N. I. et al. Human monoclonal antibodies derived from pleural effusion lymphocytes of a patient with breast carcinoma react with human breast cancer-associated antigens and mouse mammary tumor virus polypeptides. Exp. Cell Biol. 55, 237-249 (1987).

54. Kryukova, I. N., Malivanova, T. F., Kondrashova, O. M., Polevaya, E. B. \& Evtushenko, V. V. Possible pathways of circulation of human endogenous retrovirus similar to mouse mammary tumor virus. Bull Exper. Biol. Med. 120, 1176-1178 (1995).

55. Stewart, T., Tsai, S. C., Grayson, H., Henderson, R. \& Opelz, G. Incidence of de-novo breast cancer in women chronically immunosuppressed after organ transplantation. Lancet 346, 796-798 (1995).

56. Grulich, A. E. \& Vajdic, C. M. The epidemiology of cancers in human immunodeficiency virus infection and after organ transplantation. Semin. Oncol. 42, 247-257 (2015).

57. Lawson, J. S. et al. Presence of mouse mammary tumour-like virus gene sequences may be associated with specific human breast cancer morphology. J. Clin. Pathol. 59, 1287-1292 (2006).

58. Papiernik, M., Waché, A. C., Pontoux, C. \& Nabarra, B. Massive mammary gland infection and pregnancy-dependent mammary tumor development in mice infected neonatally with mouse mammary tumor virus (SW) but not in mice infected as adults, despite a dramatic local response. Eur. J. Immunol. 27, 2145-2151 (1997).

59. Hollern, D. P., Swiatnicki, M. R. \& Andrechek, E. R. Histological subtypes of mouse mammary tumors reveal conserved relationships to human cancers. PLoS Genet. 14, e1007135 (2018).

60. Fine, D. L., Arthur, L. O. \& Gardner, M. B. Prevalence of murine mammary tumor virus antibody and antigens in normal and tumor-bearing feral mice. J. Natl. Cancer Inst. 61, 485-491 (1978).

61. Day, N. K. et al. Antibodies reactive with murine mammary tumor virus in sera of patients with breast cancer: geographic and family studies. Proc. Natl Acad. Sci. USA 78, 2483-2487 (1981).

62. Briand, P. Hormone-dependent mammary tumors in mice and rats as a model for human breast cancer. Anticancer Res. 3, 273-281 (1983).

63. Wang, Y. et al. High prevalence of MMTV-like env gene sequences in gestational breast cancer. Med. Oncol. 20, 233-236 (2003).

64. Lawson, J. S. et al. Mouse mammary tumor virus-like sequences in human breast cancer. Cancer Res. 70, 3576-3585 (2010).
65. Etkind, P. R., Stewart, A. F. \& Wiernik, P. H. Mouse mammary tumor virus (MMTV)like DNA sequences in the breast tumors of father, mother, and daughter. Infect. Agent Cancer 3, 2 (2008).

66. Laumbacher, B., Fellerhoff, Herzberger, B. \& Wank, R. Do dogs harbour risk factors for human breast cancer? Med. Hypotheses 67, 21-26 (2006).

67. US Food and Drug Administration. The Food Defect Action Levels. Handbook: (2005).

68. Monot, M. et al. Early steps of Jaagsiekte sheep retrovirus-mediated cell transformation involve the interaction between Env and the RALBP1 cellular protein. $J$. Virol. 89, 8462-8473 (2015).

69. Okeoma, C. M., Huegel, A. L., Lingappa, J., Feldman, M. D. \& Ross, S. R. APOBEC3 proteins expressed in mammary epithelial cells are packaged into retroviruses and can restrict transmission of milk-borne virions. Cell Host Microbe 8, 534-543 (2010).

70. Burns, M. B. et al. APOBEC3B is an enzymatic source of mutation in breast cancer. Nature 494, 366-370 (2013).

71. Ohba, K. et al. In vivo and in vitro studies suggest a possible involvement of HPV infection in the early stage of breast carcinogenesis via APOBEC3B induction. PLOS ONE 9, e97787 (2014).

72. Vieira, V. C. et al. Human papillomavirus E6 triggers upregulation of the antiviral and cancer genomic DNA deaminase APOBEC3B. MBio. 5: e02234-2214 (2014).

73. Lawson, J. S., Salmons, B. \& Glen, W. K. Oncogenic viruses and breast cancer. Front. Oncol. 8, 1 (2018).

74. Makielsk, K. R. et al. Human papillomavirus promotes Epstein-Barr virus maintenance and lytic reactivation in immortalized oral keratinocytes. Virology 495 52-62 (2016)

75. Braitbard, O. et al. A new immunization and treatment strategy for mouse mammary tumor virus (MMTV) associated cancers. Oncotarget 7, 21168-21180 (2016).

76. Mesa-Tejada, R. et al. Detection in human breast carcinomas of an antigen immunologically related to a group-specific antigen of mouse mammary tumor virus. Proc. Natl Acad. Sci. USA 75, 1529-1533 (1978).

77. Etkind, P., Du, J., Khan, A., Pillitteri, J. \& Wiernik, P. H. Mouse mammary tumor virus-like ENV gene sequences in human breast tumors and in a lymphoma of a breast cancer patient. Clin. Cancer Res. 6, 1273-1278 (2000).

78. Melana, S. M., Holland, J. F. \& Pogo, B. G. Search for mouse mammary tumor viruslike env sequences in cancer and normal breast from the same individuals. Clin. Cancer Res. 7, 283-284 (2001).

79. Melana, S. M. et al. Detection of murine mammary tumor virus (MMTV) env genelike sequences in breast cancer from Argentine patients. Med. (B Aires). 62 323-327 (2002). (Spanish).

80. Ford, C. E., Faedo, M., Crouch, R., Lawson, J. S. \& Rawlinson, W. D. Progression from normal breast pathology to breast cancer is associated with increasing prevalence of mouse mammary tumor virus-like sequences in men and women. Cancer Res. 64, 4755-4759 (2004).

81. Zammarchi, F. et al. MMTV-like sequences in human breast cancer: a fluorescent PCR/laser microdissection approach. J. Pathol. 209, 436 (2006).

82. Hachana, M. et al. Prevalence and characteristics of the MMTV-like associated breast carcinomas in Tunisia. Cancer Lett. 271, 222-230 (2008).

83. Glenn, W. K. et al. Epstein-Barr virus, human papillomavirus and mouse mammary tumour virus as multiple viruses in breast cancer. PLOS ONE 7, e48788 (2012).

84. Slaoui, M. et al. Detection of MMTV-Like sequences in Moroccan breast cancer cases. Infect. Agent Cancer 9, 37 (2014).

85. Naushad, W. et al. Detection and identification of mouse mammary tumor viruslike DNA sequences in blood and breast tissues of breast cancer patients. Tumour Biol. 35, 8077-8086 (2014).

86. Shariatpanahi, S., Farahani, N., Salehi, A. R. \& Salehi, R. High prevalence of mouse mammary tumor virus-like gene sequences in breast cancer samples of Iranian women. Nucleosides Nucleotides Nucleic Acids 36, 621-630 (2017).

87. Al Dossary, R., Alkharsah, K. R. \& Kussaibi, H. Prevalence of Mouse Mammary Tumor Virus (MMTV)-like sequences in human breast cancer tissues and adjacent normal breast tissues in Saudi Arabia. BMC Cancer 18, 170 (2018).

88. Seo, et al. Clinical and prognostic value of human mammary tumor virus in Korean patients with breast carcinoma. Ann. Clin. Lab Sci. 49, 171-174 (2019).

89. Müller, M. \& Grossman, H. An antigen in human breast cancer sera related to the murine mammary tumour virus. Nat. New Biol. 237, 116-117 (1972).

90. Ogawa, H. \& Tanaka, H. Occurrence of antibody against intracytoplasmic Aparticles of mouse mammary tumor virus in sera from breast cancer patients. Gan 69, 539-544 (1978).

91. Mehta, S. P., Sirsat, S. M. \& Jussawalla, D. J. Humoral antibodies to mouse mammary tumor virus in sera from breast cancer patients. Indian J. Exp. Biol. 16, 1126-1130 (1978).

92. Witkin, S. S., Egeli, R. A., Sarkar, N. H., Good, R. A. \& Day, N. K. Virolysis of mouse mammary tumor virus by sera from breast cancer patients. Proc. Natl Acad. Sci. USA 76, 2984-2987 (1979). 
93. Imai, M., Yamada, C., Saga, S., Nagayoshi, S. \& Hoshino, M. Immunological cross reaction between sera from patients with breast cancer and mouse mammary tumor virus. Gan 70, 63-74 (1979).

94. Witkin, S. S., Sarkar, N. H., Kinne, D. W., Good, R. A. \& Day, N. K. Antibodies reactive with the mouse mammary tumor virus in sera of breast cancer patients. Int $J$. Cancer 25, 721-725 (1980).

95. Nagayoshi, S. et al. Use of the immune adherence hemagglutination test for titration of breast cancer patients' sea cross-reacting with purified mouse mammary tumor virus. Gan 72, 98-103 (1981).

96. Tomana, M., Kajdos, A. H., Niedermeier, W., Durkin, W. J. \& Mestecky, J. Antibodies to mouse mammary tumor virus-related antigen in sera of patients with breast carcinoma. Cancer 47, 2696-2703 (1981).

97. Zotter, S., Grossmann, H., Johannsen, B. A. \& Pilz, C. Is there any diagnostic relevance of human antibodies which react with mouse mammary tumor virus (MuMTV)? Arch. Geschwulstforsch. 51, 338-343 (1981).

98. Litvinov, S. V., Remennik, I. V. \& Kriukova, I. N. [Humoral antibodies to the antigens related to the structural protein antigens of the mammary cancer virus (MTV) in breast cancer patients and in healthy donors]. Biull Eksp. Biol. Med. 97, 600-603 (1984). Russian.

99. Chattopadhyay, J., Chattopadhyay, U. \& Chowdhury, J. R. Immunological relatedness of a murine mammary tumor-associated antigen and human breast cancer. Gan 75, 342-348 (1984).

\section{ACKNOWLEDGEMENTS}

Generoso Bevilacqua, Brian Salmons and Walter Gunzburg gave valuable assistance with the review. Desiree Berry gave advice regarding serology. Vladimir Sytnyk translated Russian articles. Stanislav Indik and Tilmann Schneider helped prepare figures.

\section{AUTHOR CONTRIBUTIONS}

J.S.L.: Development of concepts, participation in workshop on MMTV and human breast cancer, review of the literature, preparation and writing the manuscript.
W.K.G.: Development of concepts, review of the literature, preparation and writing the manuscript.

\section{COMPETING INTERESTS}

The authors declare no competing interests.

\section{ADDITIONAL INFORMATION}

Correspondence and requests for materials should be addressed to J.S.L.

Reprints and permission information is available at http://www.nature.com/ reprints

Publisher's note Springer Nature remains neutral with regard to jurisdictional claims in published maps and institutional affiliations.

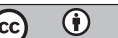

Open Access This article is licensed under a Creative Commons Attribution 4.0 International License, which permits use, sharing, adaptation, distribution and reproduction in any medium or format, as long as you give appropriate credit to the original author(s) and the source, provide a link to the Creative Commons license, and indicate if changes were made. The images or other third party material in this article are included in the article's Creative Commons license, unless indicated otherwise in a credit line to the material. If material is not included in the article's Creative Commons license and your intended use is not permitted by statutory regulation or exceeds the permitted use, you will need to obtain permission directly from the copyright holder. To view a copy of this license, visit http://creativecommons. org/licenses/by/4.0/.

(c) The Author(s) 2019 